\title{
Loss Of Heterozygosity (LOH)
}

National Cancer Institute ( $\mathrm{NCl})$

\section{Source}

National Cancer Institute (NCI). Loss of heterozygosity.

If there is one normal and one abnormal allele at a particular locus, as might be seen in an inherited autosomal dominant cancer susceptibility disorder, loss of the normal allele produces a locus with no normal function. When the loss of heterozygosity involves the normal allele, it creates a cell that is more likely to show malignant growth if the altered gene is a tumor suppressor gene. Also called LOH. 